\title{
Perceptive Packet Scheduling for Carrier Aggregation in Satellite Communication Systems
}

\author{
Hayder Al-Hraishawi, Nicola Maturo, Eva Lagunas, and Symeon Chatzinotas \\ Interdisciplinary Centre for Security, Reliability and Trust (SnT), University of Luxembourg. \\ emails: \{hayder.al-hraishawi, nicola.maturo, eva.lagunas, symeon.chatzinotas\}@uni.lu
}

\begin{abstract}
Carrier Aggregation is one of the essential approaches to achieve several orders of magnitude increase in data rates. While carrier aggregation benefits have been extensively studied in terrestrial wireless systems, its application to satellite has not been substantially explored. Carrier aggregation can be a prominent solution to address the issue of the continuously growing satellite data traffic demand. This paper studies introducing carrier aggregation in satellite systems from link layer perspective. However, deployment of carrier aggregation in satellite systems with the combination of multiple carriers that have different characteristics requires effective scheduling schemes for reliable communications. Since channel awareness is indispensable for any efficient resource allocation schemes, we have proposed a perceptive scheduling algorithm that takes into account channel properties along with the instantaneous available resources to ensure that the received data packets are completely delivered with their exact transmission order. Simulation results are given to validate our analysis and demonstrate the design tradeoffs. Additionally, our results provide useful insights to the practical scheduler design for future satellite systems.
\end{abstract}

\section{INTRODUCTION}

Recently, satellite communication has become an outgrowth of the ever-increasing traffic demand for high throughput transmission and ubiquitous broadband coverage owing to its seamless and high speed connectivity. The ability to provide telecommunication services in a wide range of sectors such as aeronautical, maritime, military, rescue and disaster relief has led to a dramatic increase in the demand for satellite data traffic [1]. Moreover, in many emerging applications for future wireless systems such as interactive multimedia services, distributed IoT networks, and content delivery networks (CDNs), satellite technology can support this expansion, and thus, contribute to $5 \mathrm{G}$ and beyond ecosystems toward highly reliable networks with global mobile coverage [2].

The combination of enormous traffic demand with higher quality of service requirements and the scarcity of available satellite spectrum is a crucial challenge for next generation satellite communication systems. Thus, system resources have to be efficiently and thoroughly utilized in high-throughput satellites (HTSs) in order to enable large-capacity communication and to facilitate the integration with terrestrial infrastructures. Many significant contributions to the developments of resource allocation have been conducted in this context aiming at enhancing satellite system capacity. In [3], an optimal dynamic capacity allocation scheme is proposed by utilizing smart gateway diversity setup and considering user requested capacity and gateway offered capacity to minimize the losses and rate matching performance metrics.
The fundamental satellite network parameters such as uplink and downlink antenna gains, user receiving gains and noise temperature, path losses, and data rates have been jointly optimized in [4] to increase system resource utilization. Reference [5] studies system resource allocation in the forward link of multibeam satellite networks and proposes an algorithm for resource allocation with objective of satisfying the requested traffic across different beams with taking fairness into consideration. The authors in [6] have particularly studied the concept of beam-hopping to harness the benefits of flexible system architecture in order to fulfill inconstant traffic demands over time and geographical locations. Beam-hopping system allows sharing in time, power, and frequency resources among multiple beams to offer higher usable throughput.

Furthermore, carrier aggregation technique has been introduced for cellular networks in the Long Term EvolutionAdvance (LTE-A) standard to allow multiple component carriers across the available spectrum bands to be flexibly aggregated to support wider transmission bandwidth, and thus, increasing the system throughput and the overall capacity [7]. Carrier aggregation in cellular networks has achieved a considerable enhancement in performance through maximizing the spectrum utilization and satisfying the extremely high throughput requirements. Interestingly, carrier aggregation does not only efficiently exploit the available spectrum and increase system capacity but it also maintains the users' quality of service through effective interference management and avoidance capabilities [8]. Therefore, a natural step would be to combine carrier aggregation with satellite system architectures in synergy to harness the multiplexing gain by distributing the traffic dynamically over multiple carriers.

Notwithstanding that the deployment of carrier aggregation technique in cellular networks has been widely investigated, its integration in satellite communication systems has not attracted a worthwhile attention in academia. Meanwhile, the European Space Agency (ESA) has funded a project titled as CADSAT [9] that deals with introducing carrier aggregation in satellite systems, where multiple potential scenarios have been extensively explored and analyzed based on market, business and technical feasibility standpoints. The work in [10] focuses on one of the investigated scenarios, that is, multibeam multicarrier geostationary earth orbit (GEO) satellite system. Specifically, both inter-transponder and intra-transponder have been considered at the satellite payload level of the communication stack to address the difficulty of carrier-user assignment 
in an environment of multiple users that can be multiplexed in each carrier.

On the other hand, channel bonding for satellite systems has been already deployed and defined in DVB-S2X standard [11] as a scheme where a single data stream is transmitted on different RF channels through different transponders lying in the same frequency band. In contrast, carrier aggregation refers to simultaneously combining multiple contiguous and noncontiguous carriers in different spectrum bands to constitute a larger transmission bandwidth. Comparing to carrier aggregation, channel bonding has numerous immanent limitations for broadband applications that would hinder resource allocation flexibility. Moreover, channel bonding utilizes constant modulation and coding (MODCOD) schemes, where all the services employ same coding and modulation procedures, which is a very deterrent factor for its deploying in future broadband applications. These limitations have motivated this work to consider carrier aggregation in order to jointly enhance system throughput and flexibility.

Alongside with introducing carrier aggregation in satellite systems, resource allocation module has to be more flexible while scheduling radio resources for users. Besides, the assignment of carriers has to be tailored with available resources and channel characteristics of each user because it has a significant impact on network performance. Specifically, in Medium Access Control (MAC) layer, a scheduler has to be carefully designed for load balancing and Protocol Data Unit (PDU) scheduling among the aggregated carriers. More importantly, an appropriate PDU scheduling algorithm is a prime part of carrier aggregation mechanism that takes into consideration the quality of service requirements of each user and attains a certain level of fairness, and hence, leads to an enhancement in the achievable system capacity and service performance.

In this paper, we focus on establishing an architecture with a detail design for embedding carrier aggregation in satellite systems from link layer viewpoint. In other words, we will demonstrate the main essential blocks that enable carrier aggregation, namely the PDU scheduler at the gateway, and the traffic merging unit at the user terminal. The proposed structure is mainly aiming at focusing the implementation efforts on the gateway side, so that the user terminal stays as simple as possible with minimum changes required to support carrier aggregation. To this end, the traffic merging block at the user terminal is simply a First-In First-Out (FIFO) system, and hence, it is of extremely importance that the PDU scheduler module at the gateway side is perceptively distributing the incoming PDUs among the aggregated carriers to ensure that they can be easily merged in a single stream with a simple FIFO buffer such that the reconstructed PDUs at the user terminal are in the correct order with no missing PDU. Therefore, we will mainly concentrate our investigating and designing efforts in this work on a scenario where a certain user is aggregating two carriers, and then, the extension for including more carriers/users can be readily conducted based on these guidelines.
Contributions: Our distinct technical contributions can be summarized as follows:

1) Design guidelines are provided for deploying carrier aggregation technique in satellite communication systems. Specifically, the required modifications at the MAC layer of the system architecture have been studied, and accordingly, the main critical blocks are thoroughly analyzed and efficiently designed.

2) Load balancing and perceptive PDU schedulers have been jointly developed through taking into consideration channel characteristics and the instant accessible resources in order to not only achieve intelligent distribution but also to guarantee the transmitted PDUs are received in a correct order without losing any packet.

3) The performance of the proposed scheduling algorithms has been investigated in terms of achievable peak data rate and the in-order delivery success rate. Simulation results including some performance comparisons are provided to demonstrate the validity and gains of the proposed algorithms.

The rest of the paper is structured as follows. The considered system model is presented in Section II. Next, a detailed description and presentation of the proposed scheduling algorithms are provided in Section III. Numerical examples and demonstrations of various simulation results are given in section IV. Finally, conclusions are drawn in Section V.

\section{SYSTEM MODEL}

We consider the forward link of a GEO multibeam satellite system that employs multi-carrier transponders. Specifically, the focus of this work is to design the two main critical blocks that required to deploy carrier aggregation technique in satellite systems, namely packet (PDU) scheduler block at the gateway and traffic merging procedure at the user terminal. For this objective, the process of establishing carrier aggregation in a single user is thoroughly and elaborately analyzed while the presence of other users within Base Band Frames (BBFrames) is not neglected. Thus, we have introduced a parameter that determines the percentage of the BBFrame data field that can be utilized by the user for transmission, which is called fill rate and denoted as $f_{r}$. Fig. 1 depicts an illustration for the fill rate in a BBFrame.

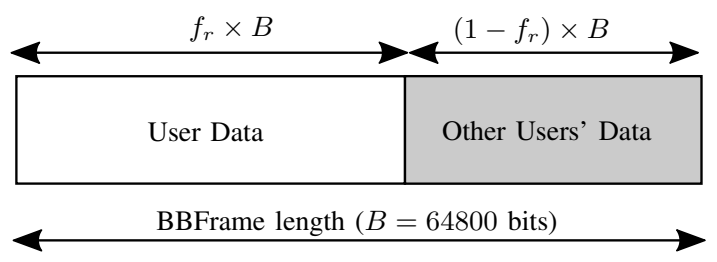

Fig. 1. User fill rate representation in BBFrame structure.

In this system, two carriers are aggregated to provide a wider bandwidth for a single user. Fig. 2 shows the changes required at the MAC layer protocol so as to ensure compatibility with Digital Video Broadcasting (DVB) (DVB-S2 and 


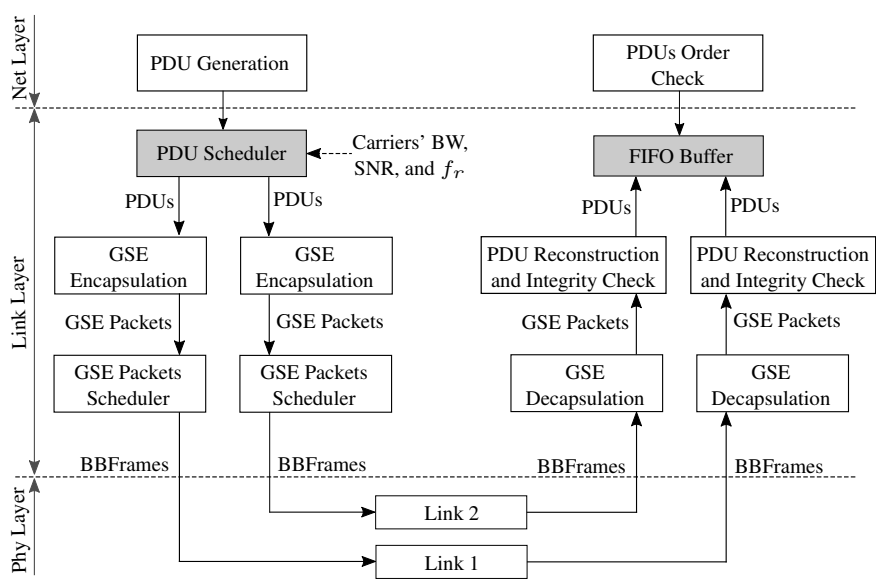

Fig. 2. Carrier aggregation link layer design.

DVB-S2X) specifications. As the link layer is the core of this system, the functionality of network layer at the transmitter side is simply represented by a PDU generator. Hence, the introduced PDU scheduler block takes the generated PDUs as input together with the parameters of the aggregated carriers, i.e. bandwidth (BW) and Signal-to-Noise Ratio (SNR), to bring forth two separated PDU streams, one for each carrier. In addition to BW and SNR, fill rate factor is also taken into consideration as another input parameter to the PDU scheduler because it reflects the actual available capacity offered to the user by each carrier individually, and thus, the dimension of the Generic Stream Encapsulation (GSE) packets will be changed accordingly.

The function of PDU scheduler is to distribute the incoming PDUs from the upper layer between the two carriers according to their channel characteristics. Afterwards, each PDU flow goes to a typical GSE encapsulation block. In this function, PDUs are encapsulated in GSE packets and according to their dimension, they could be encapsulated in a single GSE packet or sliced into PDU fragments and encapsulated in several GSE packets. Since the maximum dimension of a GSE packet is limited to $4 \mathrm{~KB}$, while the maximum dimension of a PDU can be up to 65536 bytes [12], PDU fragmentation might be required before the encapsulation. The encapsulated packets for each carrier separately are then placed together to create two streams of BBFrames. Whereas, at the link layer of the receiver side, conventional GSE decapsulation is performed and then the PDUs are reconstructed independently in both links. The integrity of the reconstructed PDUs is also checked at the link layer, and then, at the network layer and after the FIFO merging, the order of received PDUs will be examined.

\section{Proposed Scheduling Algorithms}

In this section, the concept of load balancing between the aggregated carriers is first described in brief. Next, the proposed load balance scheduling algorithm is presented. Thereafter, a perceptive scheduler design is introduced to avoid the potential pitfalls of the load balancing scheduler and also to obtain the best possible link adaptation.

\section{A. Load Balancing Scheduler}

In this subsection, we propose load balancing scheduler that distributes the incoming PDUs across the aggregated carriers based on channel conditions and the available resources. To this end, the following algorithm is developed for efficient and fair scheduling that takes into account capacity and fill rate of the considered carriers. To begin with, let us define the ratio between capacities and fill rates of the aggregated carriers as the load balancing factor $(\alpha)$, which can be calculated as follows:

$$
\alpha=\frac{C_{2} f_{r, 2}}{C_{1} f_{r, 1}}, \quad \text { where } \quad C_{1} f_{r, 1} \geq C_{2} f_{r, 2}
$$

where $C_{i}$ and $f_{r, i}$ account for the capacity and fill rate of the $i$-th carrier, respectively, and $i \in\{1,2\}$. Basically, carrier capacity is determined by the bandwidth, modulation order, and code rate, which are obtained based on the SNR of the carrier and in accordance with DVB-S2 and DVB-S2X standards, specifically Table (13) in [13] and Table (20a) in [14]. Both DVB-S2 and DVB-S2X use several MODCOD schemes to take advantage of the variable transmission conditions of each carrier, and therefore feature different capacity and transmission time with each MODCOD.

In order to expedite the scheduling process and suppress any incurred delay due to PDUs assignment, the scheduling is done by using a look-up table or allocation table contains a mapping of carrier allocation sequences and its corresponding load balancing factor $(\alpha)$ values. The length of the allocation table mainly depends on the selected granularity $(\beta)$ of $\alpha$. Thus, based on a range of $\alpha$ values with a reasonable granularity, the allocation table can be generated and stored, and hence, these carrier allocation patterns are ultimately used to schedule the incoming PDUs between the aggregated carriers. The allocation table can be dynamically and instantly modified based on the application, which gives a degree of resilience to the system. The procedure to generate the allocation table is presented in Algorithm (1).

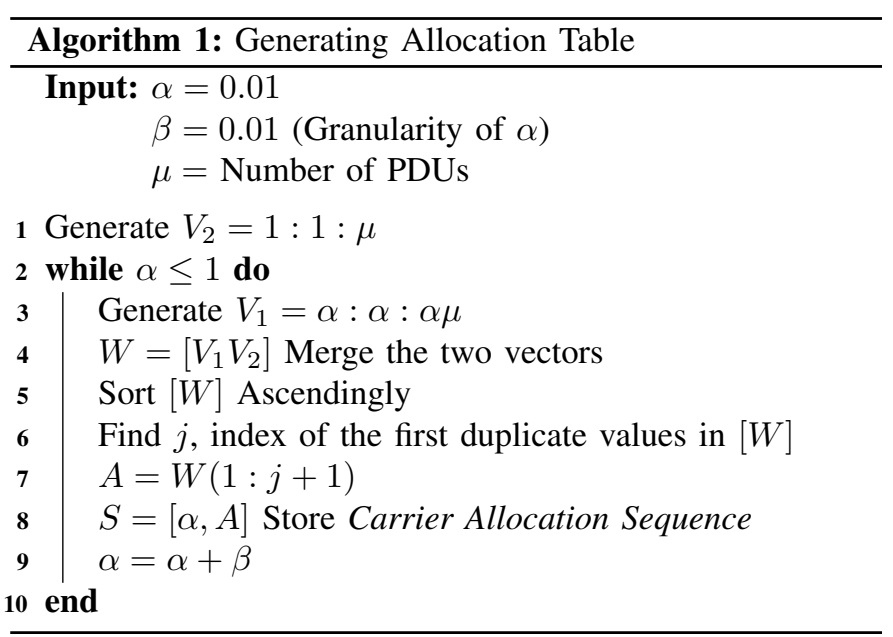

Essentially, the concept behind this algorithm is simply to prioritize sending more PDUs through the high capacity 
carrier, and vice versa. The inputs to the proposed algorithm are the number of PDUs $(\mu)$ that need to be scheduled, and the granularity $(\beta)$ of the load balancing factor $(\alpha)$. Then, two similar sequences of length $\mu, V_{1}=\alpha\{1,2, \ldots, \mu\}$ and $V_{2}=\{1,2, \ldots, \mu\}$ are generated that correspond the first and second carriers, respectively. The vector $V_{1}$ is multiply by $\alpha$, where $0<\alpha \leq 1$, in order to favour the carrier with the higher capacity and fill rate.

To further extend the design of the load balancing scheduler to deal with more than two carriers, we can follow the aforementioned methodology that we used for designing the two carriers scheduler, but we will have multiple load balancing factors (e.g. $\alpha_{1}, \alpha_{2}, \ldots$ etc.). Specifically, the number of load balancing factors equals to the number of aggregated carriers $\left(N_{C}\right)$ minus one. The carrier with highest capacity among other carriers will be the reference point to calculate the respective load balancing factors with the slower carriers. For instance, in case of aggregating three carriers $\left(N_{C}=3\right)$ the load balancing factors are calculated as follows

$$
\begin{gathered}
\alpha_{1}=\frac{C_{2} f_{r, 2}}{C_{1} f_{r, 1}}, \quad \text { and } \quad \alpha_{2}=\frac{C_{3} f_{r, 3}}{C_{1} f_{r, 1}} \\
\text { where } \quad C_{1} f_{r, 1} \geq C_{2} f_{r, 2} \geq C_{3} f_{r, 3} .
\end{gathered}
$$

Theoretically, to fully utilize the available resources in the aggregated carriers and realize an ideal load balancing, the number of PDUs that should be scheduled over each carrier has be calculated by using the following formula [15]

$$
\eta_{i}=\operatorname{round}\left(\frac{C_{i} f_{r, i}}{\sum_{j=1}^{N_{C}} C_{j} f_{r, j}} \mu\right)
$$

where $\eta_{i}$ represents the number of the assigned PDUs to the $i$-th carrier, and $N_{C}$ accounts for the number of aggregated carriers. To validate the load balancing algorithm, a comparison between the theoretical distribution and the proposed algorithm will be illustrated in the next section.

\section{B. Perceptive Scheduler}

Intuitively, scheduling PDUs to their corresponding carriers is not necessarily reflecting their transmission order in the BBFrames because they will be fragmented/encapsulated according to the assigned carrier capacity and fill rate. For instance, when a carrier has more capacity that allows to convey two PDUs or more in one BBFrame while the other carrier has space only for one PDU in a BBFrame that will bring about an ordering problem at the receiver side. Therefore, the length of the BBFrame and the instant available space in each BBFrame should be taken into consideration in the scheduling process. To this end, we have further investigated such cases and developed a supplementary procedure to track the allocation process that is done by the load balancing scheduler. The proposed procedure is conducting in advance comparison between the PDU length, the occupied bits in the current BBFrame of each carrier, and the number of transmitted BBFrames in both carriers.
Our basic idea is to incorporate the tradeoff between channel capacity and the instantaneous available resources when allocating a PDU to a carrier, and hence, do the scheduling and link adaptation collectively. In this context, BBFrame transmission is carrying out in a dynamic manner, where it has to constantly check the BBFrame creation process of the two carriers in parallel mode to verify if any further adjustment to the allocation sequence is required. Particularly, three factors are organizing and deciding this precise scheduling, (i) length of PDU, (ii) count of the transmitted BBFrames, and (iii) the remaining bits at the current BBFrame. The developed perceptive scheduling procedure is elaborated in Algorithm (2), where the occupied space in each BBFrame $\left(\psi_{i}\right)$ is updating after every transmitted PDU.

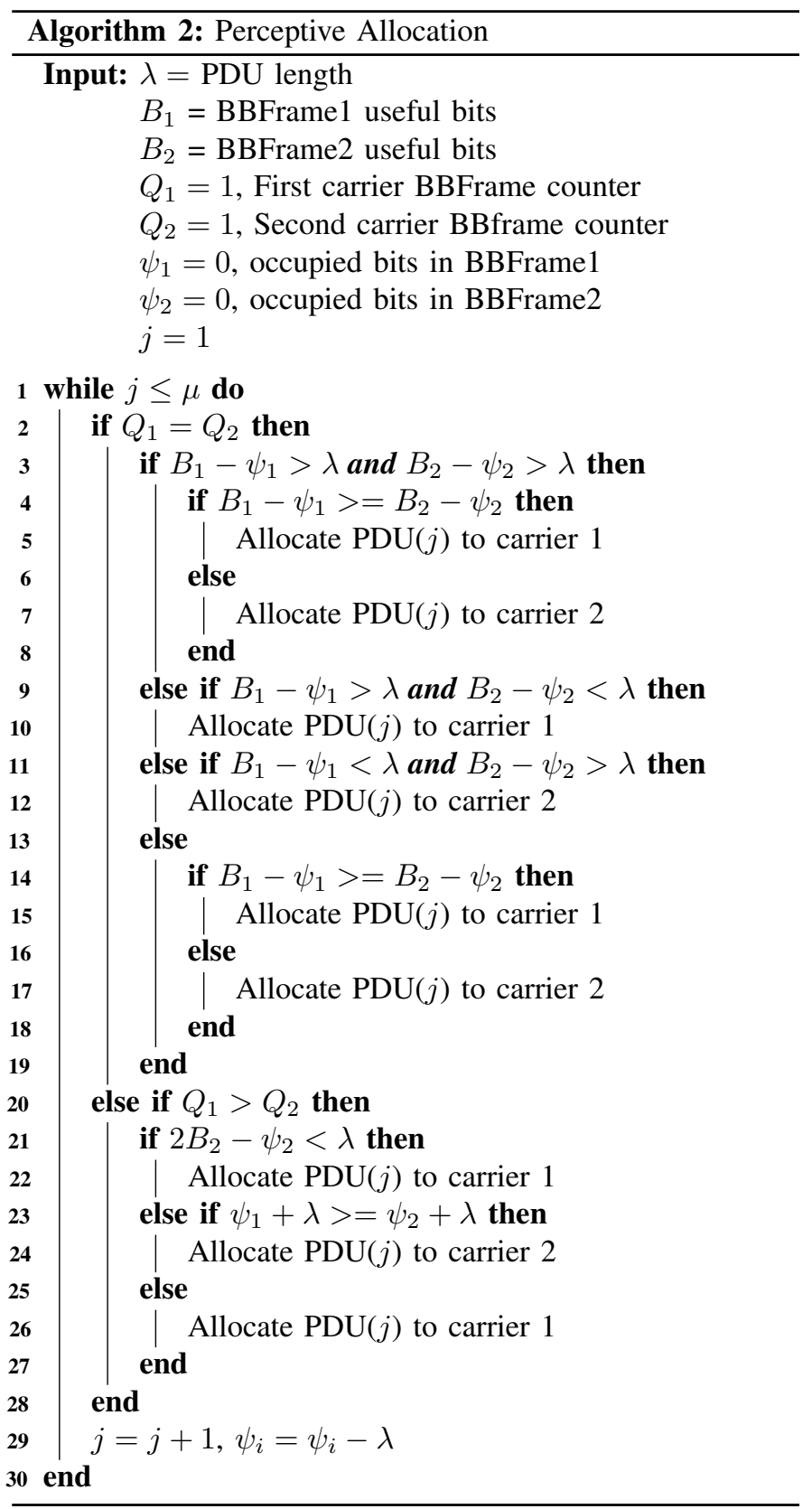


where $\lambda$ symbolizes the length of the PDUs in bits, and BBFrame useful bits account for the usable payload that a user can exploit for transmission after eliminating overheads. There are two counters tracking the number of transmitted BBFrames in each carrier and they are represented by $Q_{1}$ and $Q_{2}$, respectively. To prioritize the carrier that has sufficient space in its current BBFrame for the upcoming PDU, the factor $\psi_{i}$ is introduced in order to keep under observation the occupied space in both BBFrames. Algorithm (2) first considers the incipient phase when both carriers have equal numbers of transmitted BBFrames $\left(Q_{1}=Q_{2}\right)$, afterwards, the subsequent phases are addressed by either the case of $Q_{1}>Q_{2}$ or $Q_{1}=Q_{2}$. The case of $Q_{1}<Q_{2}$ is disregarded here because of the presumption of considering the first carrier always has the highest capacity and fill rate.

\section{Performance Evaluation}

In this section, simulation results are presented to investigate the validity and accuracy of the proposed scheduling algorithms and their performance. In Fig. 3, the achievable peak data rate of a user terminal utilizing three different carriers is scrutinized. The peak data rate is plotted against the SNR $\left(\gamma_{1}\right)$ of the first carrier, whereas the other carriers have lower SNRs. The second and third carriers have $\gamma_{2}=\gamma_{1}-3$ $\mathrm{dB}$ and $\gamma_{3}=\gamma_{1}-5 \mathrm{~dB}$, respectively. For the sake of comparison, we consider all carriers have similar bandwidth and fill rate, namely, $\mathrm{BW}=10 \mathrm{MHz}$ and $f_{r}=50 \%$. Fig. 3 shows comparisons between carrier aggregation and channel bonding from the achievable peak data rate viewpoint. It can be readily seen that carrier aggregation outperforms channel bonding in both cases of combining two carriers $\left(N_{C}=2\right)$ and three carriers $\left(N_{C}=3\right)$. Interestingly, in the useful SNR regime lower than $10 \mathrm{~dB}$, aggregating two carriers achieves slightly higher peak data rate than bonding three carriers. This observation validates the brainstorming explored in Section I, and hence, our analysis and designing insights on carrier aggregation can be useful as a benchmark for performance comparison purposes in practical high throughput satellite systems.

In Fig. 4, the performance of the proposed load balancing scheduler in Algorithm (1) is evaluated by considering two unbalanced carriers to be aggregated. The numbers of allocated PDUs to each carrier $\left(\eta_{1}\right.$ and $\left.\eta_{2}\right)$ are plotted versus the load balancing factor $(\alpha)$. Theoretical distribution curves are obtained using equation (3), and the curves of the load balancing scheduling are calculated using the sequences in the generated allocation table for each corresponding $\alpha$ value. Here we consider a stream of PDUs $(\mu=200)$ to be allotted across carrier 1 and carrier 2 of bandwidths $50 \mathrm{MHz}$ and 40 $\mathrm{MHz}$, respectively, while the SNR of the first carrier is 10 $\mathrm{dB}$ and of the second carrier is $8 \mathrm{~dB}$. Further, carriers' fill rates are setup as fixed $\left(f_{r, 1}=70 \%\right)$ for the first carrier, whilst in the second carrier varies as $f_{r, 2}=[10: 10: 100] \%$ to attain the shown $\alpha$ ranging. Fig. 4 reveals that when $\alpha$ increases the difference between $\eta_{1}$ and $\eta_{2}$ decreases till the balance point when $\alpha=1$ where both carriers convey same

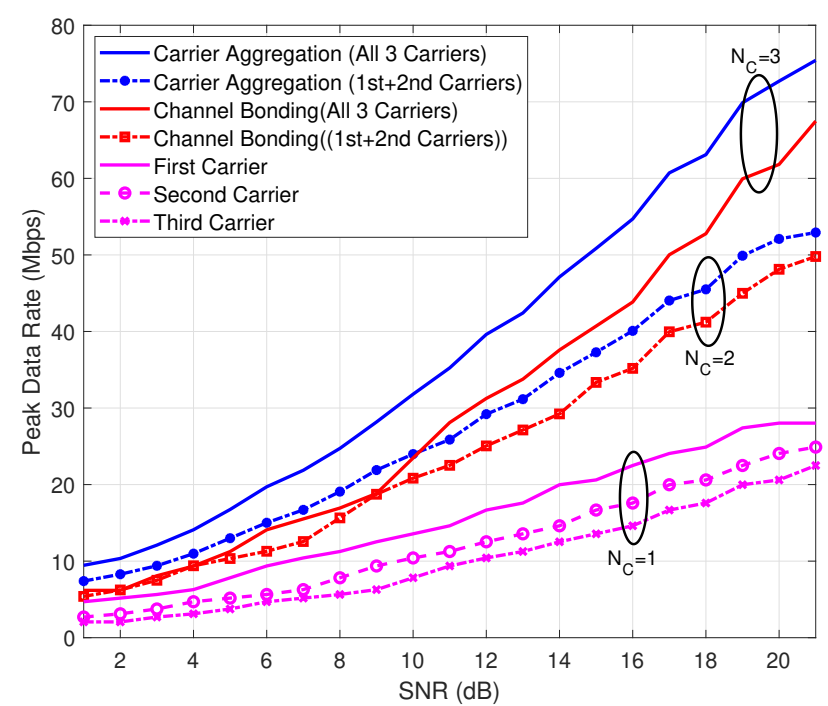

Fig. 3. The achievable peak data rate versus SNR.

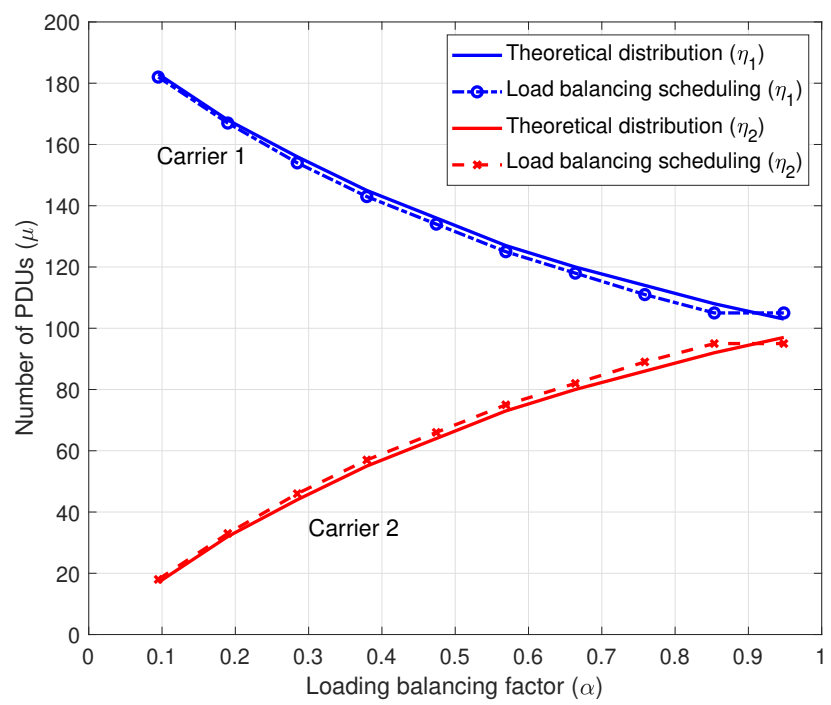

Fig. 4. Load balancing scheduler validation.

number of PDUs, i.e. $\eta_{1}=\eta_{2}$. More importantly, we can clearly observed that our algorithm is significantly tight to the theoretical distribution. Additionally, the proposed algorithm provides the actual chronological sequence for transmission.

The performance of the developed schedulers is evaluated for delivering the transmitted PDUs in a correct order. The metric used here is the ratio between the in-order received PDUs and the total number of the transmitted PDUs. In addition to the proposed schedulers, we consider Round-Robin scheduler [16] for comparison purposes. Fig. 5 presents the success rate curves of delivering in-order PDUs versus $\alpha$ by employing the aforementioned schedulers with carrier aggregation and channel bonding techniques. Two carriers are considered with bandwidths $50 \mathrm{MHz}$ and $40 \mathrm{MHz}$, respectively, and their SNRs are $10 \mathrm{~dB}$ and $8 \mathrm{~dB}$, respectively. Further, we fixed the fill rate of the first carrier to $70 \%$, while the fill rate of the second carrier is ranging between $f_{r, 2}=[30,100] \%$. A 100 PDUs of length 1400 bytes are processed through this 


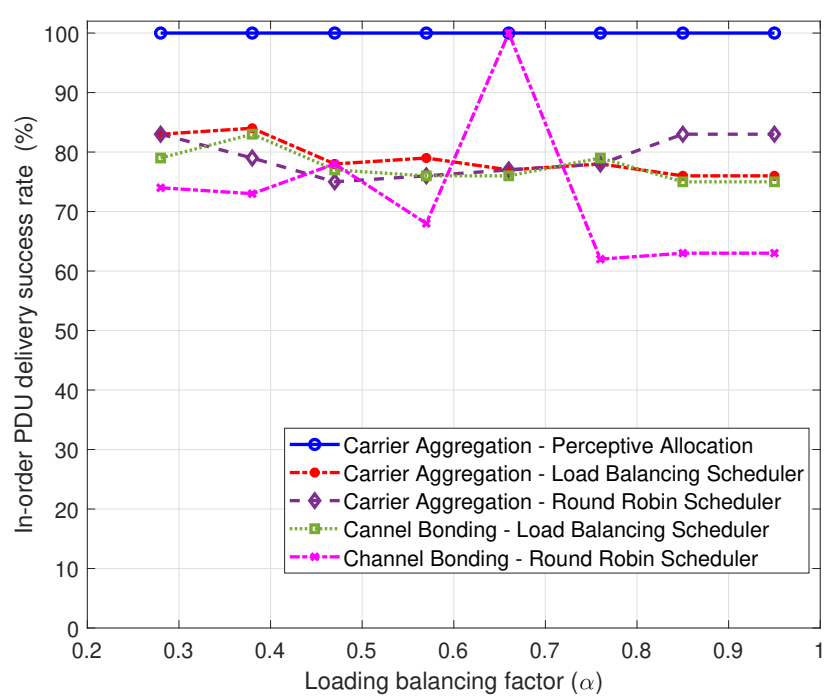

Fig. 5. In-order PDU delivery success rate versus $\alpha$.

simulation via implemented GSE encapsulation function at the transmitter side that is constructed based on conventional GSE protocol [12]. Here, GSE encapsulation function takes care of PDU fragmentation and the encapsulation to constitute GSE packets. On the other side, a GSE decapsulator is processing the received BBFrames to extract the GSE packets and remove headers and trailers. Next, the extracted GSE packets are passed to the PDU reconstruction block. In case of fragmented PDUs, the CRC (Cyclic Redundancy Check) and all the elements needed to recalculate the CRC are passed as well.

Fig. 5 clearly reveals that our perceptive scheduler in Algorithm (2) for carrier aggregation achieves the highest success rate with zero out-of-order PDU. It can also be seen that however the load balancing scheduler distributes PDUs efficiently between the considered carriers but it is not enough by itself to deliver them in-order at the receiver side. Similarly, Round-Robin scheduler cannot guarantee the order of the received PDUs unless both carriers are balanced because it is neither considering the available spectrum resources nor tracking the BBFrame creation process. Further, it can be concluded that applying channel bonding to broadband satellite systems requires more efficient and complicated schedulers to avoid the ordering issue. Therefore, the proposed perceptive scheduling algorithm serves as an efficient mean for carrier aggregation that can be used in designing practical satellite systems.

\section{CONCLUSiOns}

This paper constructed a new link layer structure to integrate carrier aggregation technique into satellite systems, and proposed data packet scheduling algorithms to efficiently utilize the considered carriers. The proposed load balancing scheduler allocates the incoming packets based on link capacity and fill rate of the aggregated carriers. This scheduler provides fair allocation to the transmitted packets and efficiently utilizes carriers' resources but it brings about an ordering issue at the receiver. Thus, it can be beneficial for some applications that have high tolerance to out-of-order delivery. To address the ordering challenge, perceptive scheduling algorithm has been developed that incorporates the tradeoff between channel capacity and the instantaneous availability of the spectral resources. The latter scheduler outperforms the proposed load balancing allocation scheme and the traditional Round-Robin scheduler in terms of in-order packet delivery.

\section{ACKNOWLEDGEMENT}

This work has received funding from the European Space Agency (ESA) funded activity CADSAT: Carrier Aggregation in Satellite Communication Networks. The views of the authors of this paper do not necessarily reflect the views of ESA.

\section{REFERENCES}

[1] S. K. Sharma, S. Chatzinotas, and P.-D. Arapoglou, Satellite Communications in the $5 G$ Era. Institution of Engineering and Technology, 2018.

[2] F. Tariq, M. R. A. Khandaker, K. Wong, M. A. Imran, M. Bennis, and M. Debbah, "A speculative study on 6G," 2019. [Online]. Available: http://arxiv.org/abs/1902.06700

[3] A. J. Roumeliotis, C. I. Kourogiorgas, and A. D. Panagopoulos, "Optimal dynamic capacity allocation for high throughput satellite communications systems," IEEE Wireless Commun. Lett., vol. 8, no. 2, pp. 596-599, Apr. 2019.

[4] J. J. Knab, "Optimization of commercial satellite transponders and terminals," IEEE Trans. Aeronaut. Navig. Electron., vol. 49, no. 1, pp. 617-622, Jan 2013.

[5] G. Cocco, T. de Cola, M. Angelone, Z. Katona, and S. Erl, "Radio resource management optimization of flexible satellite payloads for DVB-S2 systems," IEEE Trans. Broadcast., vol. 64, no. 2, pp. 266-280, Jun. 2018.

[6] C. Rohde, R. Wansch, S. Amos, H. Fenech, N. Alagha, S. Cioni, G. Mocker, and A. Trutschel-Stefan, "Beam-hopping systems for nextgeneration satellite communication systems," in Satellite Communications in the $5 G$ Era. Institution of Engineering and Technology, 2018 , ch. 10, pp. 277-305.

[7] Y. Rui, P. Cheng, M. Li, Q. T. Zhang, and M. Guizani, "Carrier aggregation for LTE-advanced: uplink multiple access and transmission enhancement features," IEEE Trans. Wireless Commun., vol. 20, no. 4, pp. 101-108, Aug. 2013.

[8] H. Lee, S. Vahid, and K. Moessner, "A survey of radio resource management for spectrum aggregation in LTE-advanced," IEEE Commun. Surveys Tuts., vol. 16, no. 2, pp. 745-760, 2014.

[9] European Space Agency (ESA), "CADSAT - Carrier aggregation in satellite communication networks," https://artes.esa.int/projects/cadsat, 2018-2020.

[10] M. Kibria, E. Lagunas, N. Maturo, D. Spano, H. Al-Hraishawi, and S. Chatzinotas, "Carrier aggregation in multi-beam high throughput satellite systems," in IEEE Global Commun. Conf., Dec. 2019.

[11] ETSI TR 102 376-2, "Digital Video Broadcasting (DVB): Implementation guidelines for the second generation system for broadcasting, interactive services, news gathering and other broadband satellite applications;part 2: S2 extensions (DVB-S2X).” European Telecommunications Standards Institute, Standard, 2015.

[12] ETSI TS 102 606-1 V1.2.1, "Digital Video Broadcasting (DVB): Generic stream encapsulation (GSE); part 1: Protocol technical specifications," European Telecommunications Standards Institute, Standard, 2014.

[13] ETSI EN 302 307-1, "Digital Video Broadcasting (DVB): Digital video broadcasting $(\mathrm{dvb})$ : Second generation framing structure, channel coding and modulation systems for broadcasting, interactive services, news gathering and other broadband satellite applications; part 1: DVB-S2." European Telecommunications Standards Institute, Standard, 2014.

[14] ETSI EN 302 307-2, "Digital Video Broadcasting (DVB): Second generation framing structure, channel coding and modulation systems for broadcasting, interactive services, news gathering and other broadband satellite applications; part 2: DVB-S2X." European Telecommunications Standards Institute, Standard, 2014.

[15] Y. Wang, K. I. Pedersen, T. B. Sorensen, and P. E. Mogensen, "Carrier load balancing and packet scheduling for multi-carrier systems," IEEE Trans. Wireless Commun., vol. 9, no. 5, pp. 1780-1789, May 2010.

[16] X. Yuan and Z. Duan, "Fair round-robin: A low complexity packet schduler with proportional and worst-case fairness," IEEE Trans. Comput., vol. 58, no. 3, pp. 365-379, March 2009. 